\title{
Uma Revisão Sistemática da Literatura sobre o uso de realidade aumentada na experiência de usuários com catálogos virtuais de produtos.
}

Dorival G. Vendrami Junior;

Berenice Gonçalves;

Marcelo Gitirana Gomes Ferreira

resumo:

Este artigo apresenta uma revisão sistemática da literatura sobre qualidade de uso de realidade aumenta na experiência de usuários com catálogos virtuais de produtos de moda nos últimos 5 anos. Os objetivos foram: apontar a curva de relevância do tema, principais periódicos e instituições que tratam da tecnologia, objetivos das pesquisas, plataformas utilizadas, tipos de simulação, modalidades de interação e tecnologias utilizadas, métodos de avaliação e as principais dificuldades. Foram catalogados 37 referências e estas demonstram o estado atual da área: o aumento de publicações no período; o Journal of Retailing and Consumer Services como grande centro ligado à área de moda, comportamento do consumidor, comunicação e tecnologia aplicada e o Lecture Notes in Computer Science, em tecnologia propriamente dita; as principais ocorrências de palavras-chave; principais pesquisadores; a dificuldade de identificação de métodos de avaliação e falta de padronização em nomenclaturas de tecnologias, entre outras descobertas.

palavras-chave:

Revisão sistemática da literatura; Realidade aumentada; Interface; Catálogos virtuais de moda 


\section{Introdução}

A busca por formas de auxiliar o usuário do comércio eletrônico na visualização de produtos antes de efetivar sua compra faz com que essa área busque constantemente novas tecnologias. A realidade aumentada, tecnologia que mistura, através de dispositivos de visualização, ambientes reais com objetos virtuais em tempo real (HÖLH, 2009), tem sido utilizada como ferramenta nos comércios eletrônicos simulando produtos de duas formas: no ambiente e no corpo do usuário (jóias, roupas, calçados etc.).

A tecnologia em si não é nova: em 1998, o termo era utilizado em suas primeiras aplicações comerciais (ROUSE, 2016), mas seu uso se deu apenas em interfaces de computadores desktop. Após um período de pouca utilização, a realidade aumentada voltou a ser vista em maior escala e frequência, com a possibilidade de auxiliar o comércio eletrônico, a realidade aumentada vem sendo utilizada por este em aplicações para o universo de produtos vestíveis (jóias, roupas, calçados e acessórios de moda) e, assim, avaliações da experiência do usuário em tais situações de uso se fazem necessárias.

A pesquisa nas principais bases de dados científicas demonstra uma quantidade relevante de artigos estudando o tema realidade aumentada em catálogos virtuais de produtos, especialmente os que projetam objetos no ambiente, além da utilização de diversas formas de avaliação das experiências nesta revisão aqui descrita, antes da aplicação dos critérios de seleção que excluíram as pesquisas com tais características, o portfólio inicial somava 2511 referências. Considerando o avanço na solução das questões técnicas e a busca de oportunidades no campo do design de publicações de catálogos digitais, este artigo apresenta uma revisão sistemática da literatura quanto à experiência do usuário no uso da realidade aumentada em catálogos virtuais de produtos de moda. Os objetivos dessa revisão são identificar principais periódicos e instituições que tratam do tema, os objetivos das pesquisas, as plataformas utilizadas, os tipos de simulação, as modalidades de interação, as tecnologias de realidade aumentada e os métodos de avaliação da tecnologia. A pesquisa pretendeu responder a questão: quais pesquisas vêm sendo realizadas no desenvolvimento de tecnologias e métodos de avaliação do uso de realidade aumentada para experiência de usuários com catálogos virtuais de produtos de vestuário?

\section{Metodologia}

Este artigo é resultado de uma Revisão Sistemática da Literatura: uma pesquisa exploratória com o objetivo de responder questões relativas a estudos realizados na construção e avaliação de experiência de realidade aumentada em catálogos virtuais de produtos vestíveis. Tal estudo será desenvolvido buscando responder as seguintes questões (Quadro 1):

Quadro 1 - Perguntas da revisão sistemática de literatura desenvolvida.

\begin{tabular}{|l|l|}
\hline Área & Pergunta \\
\hline Pesquisa reportada & Quais os principais periódicos que tratam do tema? \\
\hline & $\begin{array}{l}\text { Quais as principais instituições trabalhando no tema e seus } \\
\text { países? }\end{array}$ \\
\hline Quais os objetivos das pesquisas? \\
\hline Domínio do protótipo & Para quais plataformas os protótipos foram desenvolvidos? \\
\hline Domínio do usuário & $\begin{array}{l}\text { Quais produtos são simulados em Realidade Aumentada pelos } \\
\text { protótipos? }\end{array}$ \\
\hline & Quais as modalidades de interação usuário-protótipo? \\
\hline Quais os tipos de avaliação ergonômica da experiência com o \\
protótipo são utilizados nas pesquisas?
\end{tabular}

Fonte: acervo do autor. 
A busca desenvolvida nas bases de dados Elsevier Scopus, Science Direct, ISI Web of Science, Scielo e ProQuest, sendo que a escolha destas se deu pela abrangência, acesso ao conteúdo e presença de periódicos ligados ao tema da pesquisa. A busca compreende título, palavras-chave e resumo das publicações, nas línguas português e inglês.

Os termos da string de busca são: augmented reality; user experience, interface; evaluation, usability; virtual catalog, e-commerce, demonstration e fashion, compondo: ("augmented reality" OR "mixed reality") AND (("user experience" OR "interface") OR (evaluation OR usability)) AND (("virtual catalog" OR e-commerce OR demonstration) OR fashion).

Tais termos foram definidos por constituírem a resposta à pergunta de pesquisa, sendo:

- "augmented reality" OR "mixed reality": objeto da pesquisa.

- ("user experience" OR "interface"): o que será analisado nas aplicações. O termo "user experience" engloba a "interface", mas este é, também, utilizado isoladamente.

- (evaluation OR usability): pesquisas que avaliaram usos de realidade aumentada.

- ("virtual catalog" OR e-commerce OR demonstration) OR fashion: delimitação do uso da realidade aumentada para catálogos virtuais e para a área de moda. É utilizado OR e não AND pois a busca com este retornou uma quantidade pequena de resultados, deixando a pesquisa muito restrita.

Na seleção, foi utilizado o gerenciador Start, que permite a verificação de duplicidade e separar artigos dentro de critérios definidos. Foram aplicadas duas filtragens consecutivas:

- Filtro 1: leitura de Título, Resumo e Palavras-chave de cada artigo;

- Filtro 2: leitura completa dos artigos.

Para a segunda filtragem, foram buscados os arquivos digitais das referências selecionadas. As referências cujo obtenção por meios legais e gratuitos não foi possível, foram excluídas.

Os critérios de inclusão dos artigos extraídos com a string apresentada são:

- Pesquisas que abordem o desenvolvimento e/ou a avaliação de catálogos virtuais de produtos vestíveis que utilizam realidade aumentada;

- Artigos de periódicos publicados ou aceitos para publicação;

- Artigos de congressos publicados ou aceitos para publicação;

- Capítulos de livro;

- Teses de doutorado;

- Artigos acessíveis de forma gratuita;

- Artigos escritos em idiomas dominados pelos pesquisadores: Inglês, Português.

Critérios de exclusão:

- Pesquisas que abordem a utilização da realidade aumentada em áreas não afins à moda ou soluções que não se enquadram como catálogos virtuais;

- Artigos cuja obtenção envolva o pagamento para acesso;

- Artigos obtidos de forma cujo legalidade possa ser questionada;

- Artigos escritos em idiomas não dominados pelos pesquisadores;

- Artigos publicados há mais de 5 anos.

\section{Resultados}

As pesquisas nas bases de dados ocorreram no dia 15 de setembro de 2019. Os conjuntos de referências de cada base foram importados no programa Start, totalizando, após a exclusão de arquivos duplicados, um portfólio inicial de 2048 referências.

A primeira seleção eliminou referências que não se enquadram nos critérios da revisão a partir da leitura de título, palavras-chave, resumo, data de publicação e pela linguagem dos documentos. Desta primeira filtragem resultou uma lista de 142 artigos.

$\mathrm{Na}$ fase seguinte, a leitura diagonal dos artigos revelou que113 artigos não se enquadravam nos objetivos da revisão, deixando um total de 31 artigos para a extração dos dados. O quadro 2 demonstra os resultados e índice de aproveitamento de cada base: 
Quadro 2 - Resultado da busca nas bases de dados e filtragem.

\begin{tabular}{|c|c|c|c|c|c|c|}
\hline Base de dados & Scopus & $\begin{array}{c}\text { Science } \\
\text { Direct }\end{array}$ & $\begin{array}{c}\text { ISI Web of Sci- } \\
\text { ence }\end{array}$ & ProQuest & Scielo & Total \\
\hline $\begin{array}{c}\text { Artigos na } \\
\text { extração }\end{array}$ & 242 & 2641 & 65 & 1053 & 25 & 2511 \\
\hline $\begin{array}{c}\text { Artigos no } \\
\text { portfólio final }\end{array}$ & 10 & 13 & 1 & 7 & 0 & 31 \\
\hline Aproveitamento & $4,13 \%$ & $0,49 \%$ & $1,53 \%$ & $0,66 \%$ & $0 \%$ & $1,36 \%$ \\
\hline
\end{tabular}

Fonte: acervo do autor.

Após a seleção final, foi aplicada a técnica de snowbowlling: análise das referências e citações dos documentos selecionados e busca de pesquisas que se encaixem nos critérios que utilizamos. Desse modo, o quadro abaixo 3 demonstra as 37 referências do portfólio final desta revisão:

Quadro 3: Conjunto final de documentos resultante da Revisão Sistemática da Literatura.

\begin{tabular}{|c|c|c|}
\hline $\begin{array}{l}\text { DOI: } \\
\text { 10.1016/ } \\
\text { j.jretconser.2 } \\
016.08 .006\end{array}$ & $\begin{array}{l}\text { BECK, Marie; CRIÉ, Dominique. I virtually try it ... I want it ! } \\
\text { Virtual Fitting Room: A tool to increase on-line and off-line ex- } \\
\text { ploratory behavior, patronage and purchase intentions. Journal } \\
\text { Of Retailing And Consumer Services, [s.1.], v. 40, p.279-286, } \\
\text { jan. 2018. Elsevier BV. }\end{array}$ & $\begin{array}{l}\text { Science } \\
\text { Direct }\end{array}$ \\
\hline $\begin{array}{c}\text { DOI: } \\
10.1016 / \\
\text { j.jretconser. } 2 \\
019.101938\end{array}$ & $\begin{array}{l}\text { BONNIN, Gaël. The roles of perceived risk, attractiveness of the } \\
\text { online store and familiarity with AR in the influence of AR on } \\
\text { patronage intention. Journal Of Retailing And Consumer Ser- } \\
\text { vices, [s.1.], v. 52, p.101938-101941, jan. 2020. Elsevier BV. }\end{array}$ & $\begin{array}{l}\text { Science } \\
\text { Direct }\end{array}$ \\
\hline $\begin{array}{c}\text { ISSN: } \\
19389027\end{array}$ & $\begin{array}{l}\text { CHANDRA, Shalini; KUMAR, Karippur Nanda. EXPLORING } \\
\text { FACTORS INFLUENCING ORGANIZATIONAL ADOPTION } \\
\text { OF AUGMENTED REALITY IN E-COMMERCE: EMPIRI- } \\
\text { CAL ANALYSIS USING TECHNOLOGY-ORGANIZATION- } \\
\text { ENVIRONMENT MODEL. Journal Of Electronic Commerce } \\
\text { Research. Long Beach, p. 237-265. ago. } 2018 .\end{array}$ & $\begin{array}{l}\text { Pro- } \\
\text { Quest }\end{array}$ \\
\hline $\begin{array}{c}\text { DOI: } \\
10.1115 / 1.40 \\
42102\end{array}$ & $\begin{array}{l}\text { CHU, Chih-hsing et al. A Cloud Service Framework for Virtual } \\
\text { Try-On of Footwear in Augmented Reality. Journal Of Com- } \\
\text { puting And Information Science In Engineering, [s.1.], v. 19, } \\
\text { n. 2, p.0210021-0210027, } 4 \text { fev. 2019. ASME International. }\end{array}$ & $\begin{array}{l}\text { ISI WoS } \\
\text { e Scopus }\end{array}$ \\
\hline $\begin{array}{l}\text { DOI: } \\
10.1007 / \\
\text { s10055-018- } \\
0338-3\end{array}$ & $\begin{array}{c}\text { CRUZ, Edmanuel et al. An augmented reality application for } \\
\text { improving shopping experience in large retail stores. Virtual } \\
\text { Reality, [s.l.], v. 23, n. 3, p.281-291, } 24 \text { fev. 2018. Springer Sci- } \\
\text { ence and Business Media LLC. }\end{array}$ & $\begin{array}{l}\text { Pro- } \\
\text { Quest }\end{array}$ \\
\hline $\begin{array}{l}\text { DOI: } \\
10.1016 / \\
\text { j.techfore. } 20 \\
16.09 .032\end{array}$ & $\begin{array}{c}\text { DACKO, Scott G.. Enabling smart retail settings via mobile } \\
\text { augmented reality shopping apps. Technological Forecasting } \\
\text { And Social Change, [s.1.], v. 124, p.243-256, nov. 2017. Elsevi- } \\
\text { er BV. }\end{array}$ & $\begin{array}{c}\text { Snow- } \\
\text { bowling }\end{array}$ \\
\hline $\begin{array}{c}\text { e-ISSN: } \\
2289-8131\end{array}$ & $\begin{array}{c}\text { ELRADI, Mahgoub et al. A 3D e-Commerce Applications De- } \\
\text { velopment Model: A Systematic Literature Review. Journal Of } \\
\text { Telecommunication, Electronic And Computer Engineering. } \\
\text { Kuala Lumpur, p. 27-33. abr. } 2017 .\end{array}$ & Scopus \\
\hline
\end{tabular}


Quadro 3: Conjunto final de documentos resultante da Revisão Sistemática da Literatura (continuação).

\begin{tabular}{|c|c|c|}
\hline $\begin{array}{l}\text { DOI: } \\
10.1007 / \\
\text { s11042-018-5 } \\
758-2\end{array}$ & $\begin{array}{l}\text { ERRA, Ugo; SCANNIELLO, Giuseppe; COLONNESE, Vale- } \\
\text { rio. Exploring the effectiveness of an augmented reality dress- } \\
\text { ing room. Multimedia Tools And Applications, [s.1.], v. 77, n. } \\
\text { 19, p.25077-25107, } 20 \text { fev. 2018. Springer Science and Busi- } \\
\text { ness Media LLC. }\end{array}$ & ProQuest \\
\hline $\begin{array}{l}\text { DOI: } \\
10.1016 / \\
\text { j.procs. } 2018 \text {. } \\
04.207\end{array}$ & $\begin{array}{l}\text { FENG, Zhuming; JIANG, Fei; SHEN, Ruimin. Virtual Glasses } \\
\text { Try-on Based on Large Pose Estimation. Procedia Computer } \\
\text { Science, [s.1.], v. 131, p.226-233, 2018. Elsevier BV. }\end{array}$ & $\begin{array}{c}\text { Science } \\
\text { Direct }\end{array}$ \\
\hline $\begin{array}{l}\text { DOI: } \\
10.1007 / \\
\text { s11747-017-0 } \\
541-\mathrm{x}\end{array}$ & $\begin{array}{l}\text { HILKEN, Tim et al. Augmenting the eye of the beholder: ex- } \\
\text { ploring the strategic potential of augmented reality to enhance } \\
\text { online service experiences. Journal Of The Academy Of } \\
\text { Marketing Science, [s.1.], v. 45, n. 6, p.884-905, } 18 \text { maio } \\
\text { 2017. Springer Nature. }\end{array}$ & $\begin{array}{c}\text { Snow- } \\
\text { bowling }\end{array}$ \\
\hline $\begin{array}{l}\text { DOI: } \\
\text { 10.1108/jrim- } \\
01-2018-002 \\
3\end{array}$ & $\begin{array}{l}\text { HILKEN, Tim et al. Making omnichannel an augmented reali- } \\
\text { ty: the current and future state of the art. Journal Of Research } \\
\text { In Interactive Marketing, [s.1.], v. 12, n. 4, p.509-523, } 8 \text { out. } \\
\text { 2018. Emerald. }\end{array}$ & ProQuest \\
\hline $\begin{array}{l}\text { DOI: } \\
10.1007 / \\
\text { s10660-014- } \\
9163-2\end{array}$ & $\begin{array}{c}\text { HUANG, Tseng-lung; LIAO, Shuling. A model of acceptance } \\
\text { of augmented-reality interactive technology: the moderating } \\
\text { role of cognitive innovativeness. Electronic Commerce Re- } \\
\text { search, [s.1.], v. 15, n. 2, p.269-295, } 12 \text { nov. 2014. Springer } \\
\text { Nature. }\end{array}$ & $\begin{array}{l}\text { Snow- } \\
\text { bowling }\end{array}$ \\
\hline $\begin{array}{l}\text { DOI: } \\
\text { 10.1016/ } \\
\text { j.jretconser.2 } \\
018.11 .016\end{array}$ & $\begin{array}{l}\text { HUANG, Tseng-lung. Psychological mechanisms of brand love } \\
\text { and information technology identity in virtual retail environ- } \\
\text { ments. Journal Of Retailing And Consumer Services, [s.1.], } \\
\text { v. 47, p.251-264, mar. 2019. Elsevier BV. }\end{array}$ & $\begin{array}{c}\text { Science } \\
\text { Direct }\end{array}$ \\
\hline $\begin{array}{l}\text { DOI: } \\
10.1145 / 2901 \\
790.2901881\end{array}$ & $\begin{array}{l}\text { JAVORNIK, Ana et al. Revealing the Shopper Experience of } \\
\text { Using a 'Magic Mirror' augmented reality make-up application. } \\
\text { Proceedings Of The } \mathbf{2 0 1 6} \text { Acm Conference On Designing } \\
\text { Interactive Systems - Dis '16, [s.1.], p.1-2, 2016. ACM Press. }\end{array}$ & $\begin{array}{c}\text { Snow- } \\
\text { bowling }\end{array}$ \\
\hline $\begin{array}{l}\text { ISBN: } \\
97898604910 \\
29\end{array}$ & $\begin{array}{l}\text { KUMAR, Karippur Nanda et al. FACTORS INFLUENCING } \\
\text { ADOPTION OF AUGMENTED REALITY TECHNOLOGY } \\
\text { FOR E-COMMERCE. In: PACIS 2016: Proceedings of the } \\
\text { 20th Pacific Asia Conference on Information Systems. As- } \\
\text { sociation for Information Systems (2016). }\end{array}$ & Scopus \\
\hline $\begin{array}{l}\text { DOI: } \\
\text { 10.3390/ } \\
\text { mti1040026 }\end{array}$ & $\begin{array}{l}\text { LIBERATI, Nicola. The Emperor's New Augmented Clothes. } \\
\text { Digital Objects as Part of the Every Day. Multimodal Tech- } \\
\text { nologies And Interaction, [s.1.], v. 1, n. 4, p.26-38, } 23 \text { out. } \\
\text { 2017. MDPI AG. }\end{array}$ & ProQuest \\
\hline $\begin{array}{l}\text { DOI: } \\
10.1145 / 2964 \\
284.2970928\end{array}$ & $\begin{array}{l}\text { LIU, Yejun et al. Magic Mirror. Proceedings Of The } 2016 \\
\text { Acm On Multimedia Conference - Mm '16, [s.1.], p.680-683, } \\
\text { 2016. ACM Press. }\end{array}$ & $\begin{array}{l}\text { ISI WoS e } \\
\text { Scopus }\end{array}$ \\
\hline $\begin{array}{l}\text { DOI: } \\
10.1007 / 978- \\
3-030-20787- \\
8 \_19\end{array}$ & $\begin{array}{l}\text { MANGIARUA, Nahuel et al. Templates Framework for the } \\
\text { Augmented Catalog System. Communications In Computer } \\
\text { And Information Science, [s.1.], p.267-276, 2019. Springer } \\
\text { International Publishing. }\end{array}$ & Scopus \\
\hline
\end{tabular}


Quadro 3: Conjunto final de documentos resultante da Revisão Sistemática da Literatura (continuação).

\begin{tabular}{|c|c|c|}
\hline $\begin{array}{l}\text { DOI: } \\
10.1016 / \\
\text { j.chb. } 2019.07 \\
.002\end{array}$ & $\begin{array}{l}\text { MCLEAN, Graeme; WILSON, Alan. Shopping in the digital } \\
\text { world: Examining customer engagement through augmented reali- } \\
\text { ty mobile applications. Computers In Human Behavior, [s.1.], v. } \\
\text { 101, p.210-224, dez. 2019. Elsevier BV. }\end{array}$ & $\begin{array}{c}\text { Science } \\
\text { Direct }\end{array}$ \\
\hline & $\begin{array}{l}\text { MIELL, Sophie L.. Enabling the Digital Fashion Consumer } \\
\text { through Gamified Fit and Sizing Experience Technologies. } \\
\text { 2018. } 374 \text { f. Tese (Doutorado) - Curso de Philosophy In Textiles } \\
\text { Design, Fashion \& Management, Faculty Of Science And Engi- } \\
\text { neering, The University Of Manchester, Manchester, } 2018 .\end{array}$ & $\begin{array}{l}\text { Pro- } \\
\text { Quest }\end{array}$ \\
\hline $\begin{array}{l}\text { DOI } 10.1109 / \\
\text { ISMAR.2015 } \\
.60\end{array}$ & $\begin{array}{c}\text { OHTA, Masaya et al. [POSTER] Mixed-Reality Store on the Other } \\
\text { Side of a Tablet. 2015 Ieee International Symposium On Mixed } \\
\text { And Augmented Reality, [s.1.], p.192-193, set. 2015. IEEE. }\end{array}$ & Scopus \\
\hline $\begin{array}{c}\text { DOI: } \\
10.1145 / 3132 \\
272.3135931\end{array}$ & $\begin{array}{l}\text { OSAMURA, Kazuki et al. Proposal of Product Navigation Inter- } \\
\text { face and Evaluation of Purchasing Motivation. Proceedings Of } \\
\text { The Interactive Surfaces And Spaces On Zzz - Iss '17, [s.1.], } \\
\text { p.458-461, 2017. ACM Press. }\end{array}$ & Scopus \\
\hline $\begin{array}{l}\text { DOI: } \\
10.1016 / \\
\text { j.jretconser. } 2 \\
017.05 .011\end{array}$ & $\begin{array}{l}\text { PANTANO, Eleonora; RESE, Alexandra; BAIER, Daniel. Enhanc- } \\
\text { ing the online decision-making process by using augmented reali- } \\
\text { ty: A two country comparison of youth markets. Journal Of Re- } \\
\text { tailing And Consumer Services, [s.1.], v. 38, p.81-95, set. } 2017 . \\
\text { Elsevier BV. }\end{array}$ & $\begin{array}{c}\text { Science } \\
\text { Direct }\end{array}$ \\
\hline $\begin{array}{l}\text { DOI: } \\
\text { 10.1016/ } \\
\text { j.jretconser.2 } \\
019.07 .002\end{array}$ & $\begin{array}{l}\text { PLOTKINA, Daria; SAUREL, Hélène. Me or just like me? The } \\
\text { role of virtual try-on and physical appearance in apparel M-retail- } \\
\text { ing. Journal Of Retailing And Consumer Services, [s.1.], v. 51, } \\
\text { p.362-377, nov. 2019. Elsevier BV. }\end{array}$ & $\begin{array}{c}\text { Science } \\
\text { Direct }\end{array}$ \\
\hline $\begin{array}{l}\text { DOI: } \\
10.1016 / \\
\text { j.techfore. } 201 \\
6.10 .010\end{array}$ & $\begin{array}{l}\text { RESE, Alexandra et al. How augmented reality apps are accepted } \\
\text { by consumers: A comparative analysis using scales and opinions. } \\
\text { Technological Forecasting And Social Change, [s.1.], v. 124, } \\
\text { p.306-319, nov. } 2017 . \text { Elsevier BV. }\end{array}$ & $\begin{array}{c}\text { Science } \\
\text { Direct }\end{array}$ \\
\hline $\begin{array}{l}\text { DOI: } \\
10.1145 / 2858 \\
036.2858282\end{array}$ & $\begin{array}{l}\text { SAAKES, Daniel et al. Mirror Mirror. Proceedings Of The } 2016 \\
\text { Chi Conference On Human Factors In Computing Systems - } \\
\text { Chi '16, [s.1.], p.6058-6063, 2016. ACM Press. }\end{array}$ & $\begin{array}{c}\text { ISI } \\
\text { WoS e } \\
\text { Scopus }\end{array}$ \\
\hline $\begin{array}{l}\text { DOI: } \\
\text { 10.1016/ } \\
\text { j.jretconser.2 } \\
018.05 .004\end{array}$ & $\begin{array}{l}\text { SCHOLZ, Joachim; DUFFY, Katherine. We ARe at home: How } \\
\text { augmented reality reshapes mobile marketing and consumer-brand } \\
\text { relationships. Journal Of Retailing And Consumer Services, } \\
\text { [s.1.], v. 44, p.11-23, set. 2018. Elsevier BV. }\end{array}$ & $\begin{array}{c}\text { Science } \\
\text { Direct }\end{array}$ \\
\hline $\begin{array}{l}\text { DOI: } \\
10.1016 / \\
\text { j.bushor. } 2015 \\
.10 .003\end{array}$ & $\begin{array}{c}\text { SCHOLZ, Joachim; SMITH, Andrew N. Augmented reality: De- } \\
\text { signing immersive experiences that maximize consumer engage- } \\
\text { ment. Business Horizons, [s.1.], v. 59, n. 2, p.149-161, mar. } 2016 . \\
\text { Elsevier BV. . }\end{array}$ & $\begin{array}{l}\text { Snow- } \\
\text { bowl- } \\
\text { ing }\end{array}$ \\
\hline $\begin{array}{l}\text { DOI: } \\
\text { 10.1016/j.el- } \\
\text { erap. } 2019.10 \\
0854\end{array}$ & $\begin{array}{l}\text { SMINK, Anne R. et al. Try online before you buy: How does } \\
\text { shopping with augmented reality affect brand responses and per- } \\
\text { sonal data disclosure. Electronic Commerce Research And Ap- } \\
\text { plications, [s.1.], v. 35, p.100854-100864, maio 2019. Elsevier BV. }\end{array}$ & $\begin{array}{c}\text { Science } \\
\text { Direct }\end{array}$ \\
\hline
\end{tabular}


Quadro 3: Conjunto final de documentos resultante da Revisão Sistemática da Literatura (continuação).

\begin{tabular}{|c|c|c|}
\hline $\begin{array}{c}\text { DOI: } 10.1109 / \\
\text { inista. } 2015.72 \\
76727\end{array}$ & $\begin{array}{l}\text { STOYANOVA, Jasmina et al. Comparison of consumer purchase } \\
\text { intention between interactive and augmented reality shopping plat- } \\
\text { forms through statistical analyses. } 2015 \text { International Sympo- } \\
\text { sium On Innovations In Intelligent Systems And Applications } \\
\text { (inista), [s.1.], set. 2015. IEEE. }\end{array}$ & $\begin{array}{c}\text { Snow- } \\
\text { bowling }\end{array}$ \\
\hline $\begin{array}{c}\text { DOI: } 10.1016 / \\
\text { j.intmar.2017. } \\
04.001\end{array}$ & $\begin{array}{l}\text { YIM, Mark Yi-cheon; CHU, Shu-chuan; SAUER, Paul L.. Is } \\
\text { Augmented Reality Technology an Effective Tool for E-com- } \\
\text { merce? An Interactivity and Vividness Perspective. Journal Of } \\
\text { Interactive Marketing, [s.1.], v. 39, p.89-103, ago. 2017. Elsevier }\end{array}$ & $\begin{array}{l}\text { Science } \\
\text { Direct }\end{array}$ \\
\hline $\begin{array}{l}\text { DOI: } \\
10.1016 / \\
\text { j.promfg. } 2015 \\
.07 .766\end{array}$ & $\begin{array}{l}\text { WANG, Chao-hung; CHIANG, Yi-chen; WANG, Mao-jiun. Eval- } \\
\text { uation of an Augmented Reality Embedded On-line Shopping Sys- } \\
\text { tem. Procedia Manufacturing, [s.1.], v. 3, p.5624-5630, } 2015 . \\
\text { Elsevier BV. }\end{array}$ & $\begin{array}{l}\text { Science } \\
\text { Direct }\end{array}$ \\
\hline $\begin{array}{l}\text { DOI: } \\
10.1007 / 978-3 \\
-319-60922-5 \\
34\end{array}$ & $\begin{array}{c}\text { WELIVITA, Anuradha et al. Virtual Product Try-On Solution for } \\
\text { E-Commerce Using Mobile Augmented Reality. Lecture Notes In } \\
\text { Computer Science, [s.1.], p.438-447, 2017. Springer International } \\
\text { Publishing. }\end{array}$ & ISI WoS \\
\hline $\begin{array}{l}\text { DOI: } 10.1186 / \\
\text { s13640-018-0 } \\
373-8\end{array}$ & $\begin{array}{c}\text { ZHANG, Boping. Augmented reality virtual glasses try-on tech- } \\
\text { nology based on iOS platform. Eurasip Journal On Image And } \\
\text { Video Processing, [s.1.], v. 2018, n. 1, p.1-19, } 27 \text { nov. } 2018 . \\
\text { Springer Nature. }\end{array}$ & ProQuest \\
\hline
\end{tabular}

Fonte: acervo do autor.

\section{Discussão}

Para apresentação dos resultados, foram geradas redes com os dados dos artigos da Revisão Sistemática no aplicativo VosViewer e na base de dados Scopus. A revisão demostrou uma recorrência de pesquisas com realidade aumentada aplicada a catálogos nos últimos anos: quatro referências de 2015, cinco de 2016, dez de 2017, nove de 2018, oito de 2019 e uma de 2020 (Gráfico 1).

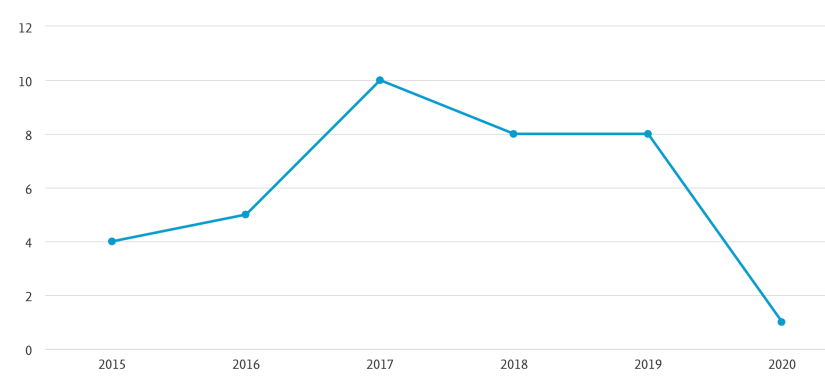

Gráfico 1: ocorrência de publicações por ano. Fonte: acervo do autor.

Os autores com maior recorrência foram Daniel Baier e Alexandra Rese, da University of Bayreuth, na Alemanha; Shalini Chandra, da S P Jain School of Global Management, nos Emirados Árabes; Mathew Chylinski, da Universidade de New South Wales, na Austrália; Tim Hilken e Dominik Mahr, da Universidade Maastricht, na Holanda; Tseng-Lung Huang, da Universidade Yuan Ze, em Taiwan; Debbie Isobel Keeling, da Universidade de Sussex, na Inglaterra; Nikunj Kumar Jain, do International Management Institute, na Índia e Joachim Scholz, da Universidade Estadual Politécnica da Califórnia, nos Estados Unidos - todos com duas publicações. Para os tipos de documento, 23 são artigos de periódicos, 12 documentos de conferências e dois são revisões de literatura (Gráfico 2). 


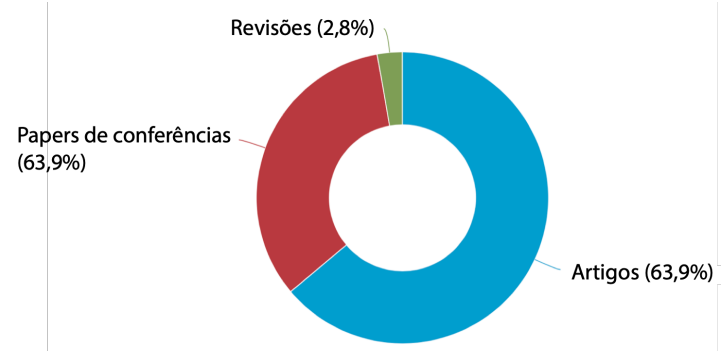

Gráfico 2- Tipos de documentos. Fonte: acervo do autor.

Quanto à nacionalidade, nove documentos foram produzidos no Reino Unido, seis nos Estados Unidos, quatro em Taiwan, sendo os demais espalhados em 17 países (Gráfico 3).

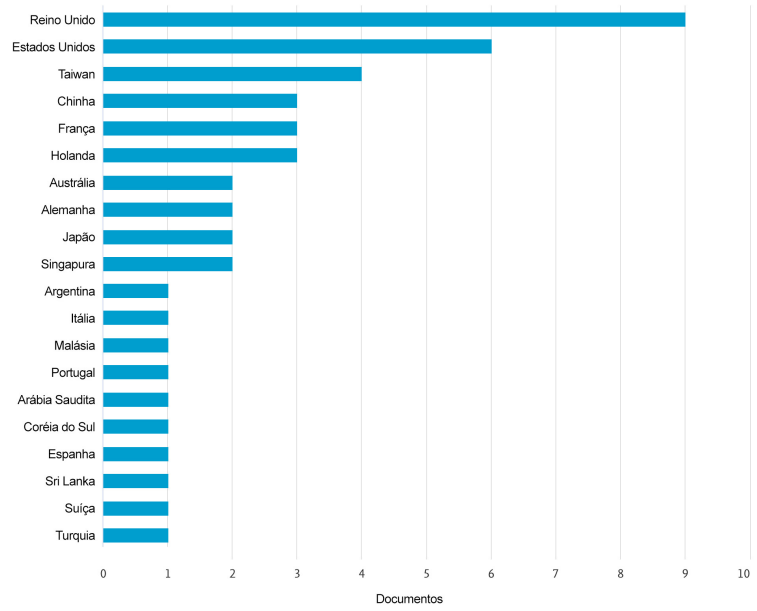

Gráfico 3 - Publicações por países. Fonte: acervo do autor.

A co-ocorrência de palavras-chave (Gráfico 4) mostra uma predominância do termo Realidade ser acompanhado por e-commerce, electronic commerce e realidade virtual. Conseguimos, ainda, distinguir quatro grupos de palavras-chave, indicando áreas em que a realidade aumentada é estudada.

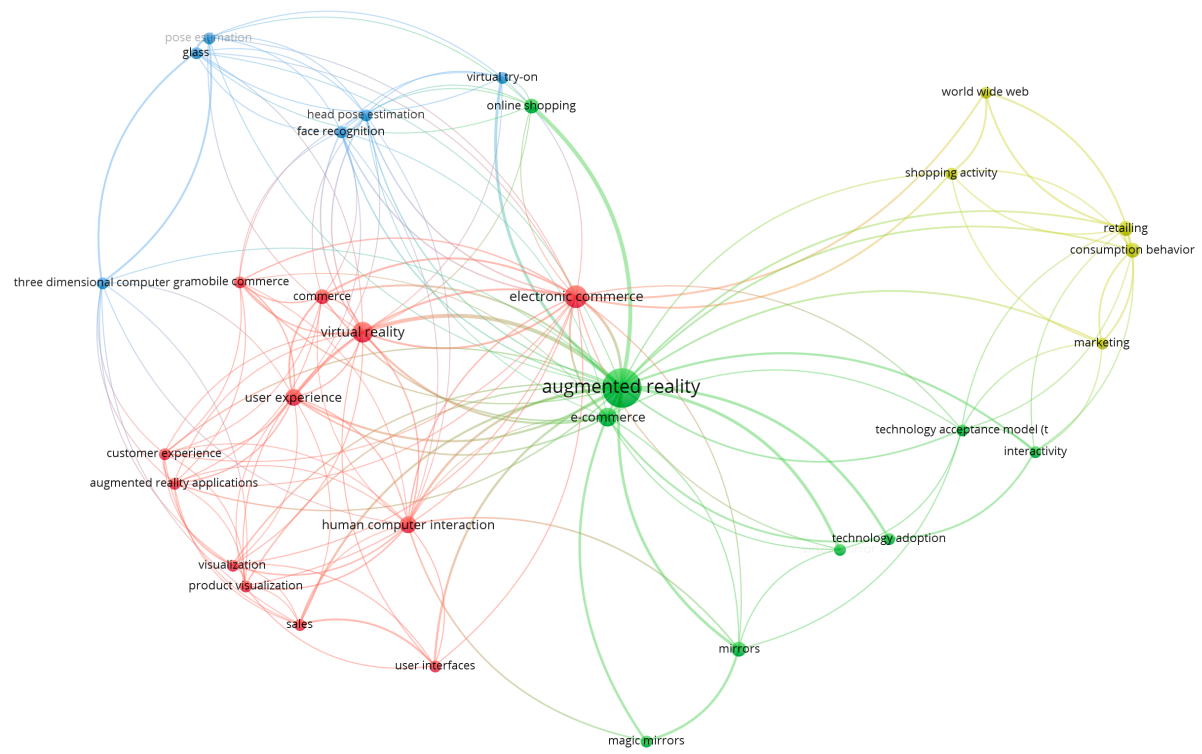

Gráfico 4 - Co-ocorrência de palavras-chave e os quatro grupos de proximidade. Fonte: acervo do autor. 
O principal grupo de palavras-chave, representado na figura 4 em verde, é formado por realidade aumentada, e-commerce, customer experience, electronic commerce, human computer interaction, mobile commerce, product visualization, sales, user experience, user interfaces, virtual reality e visualization. Já o segundo grupo, visto em vermelho na figura, consiste em realidade aumentada, ecommerce, interactivity, magic mirrors, mirrors, online shopping, technology acceptance model, technology adoption e virtual mirror. $\mathrm{O}$ terceiro grupo de palavras-chave, representado em azul, consiste em face recognition, glass, head pose estimulation, three dimensional computer graphics e virtual tryon. Por último, o quarto grupo, em amarelo: consumption behavior, marketing, retailing, shopping activity e word wide web.

O primeiro grupo sintetiza as palavras-chaves associadas ao uso da realidade aumentada de modo geral; o segundo e o terceiro enfatizam a tecnologia e o quarto é focado em marketing.

Quando avaliadas as citações por organização (Gráfico 5), pode-se identificar três grupos de interesse e estudo, marketing (vermelho), tecnologia (azul) e um cruzamento entre ambos (verde), com foco no estudo da tecnologia junto ao usuário. Além disso, é possível identificar como a questão tecnológica possui força na Alemanha, Inglaterra e avança para India e oriente, enquanto o marketing é predominantemente estudado entre Inglaterra, Estados Unidos e França.

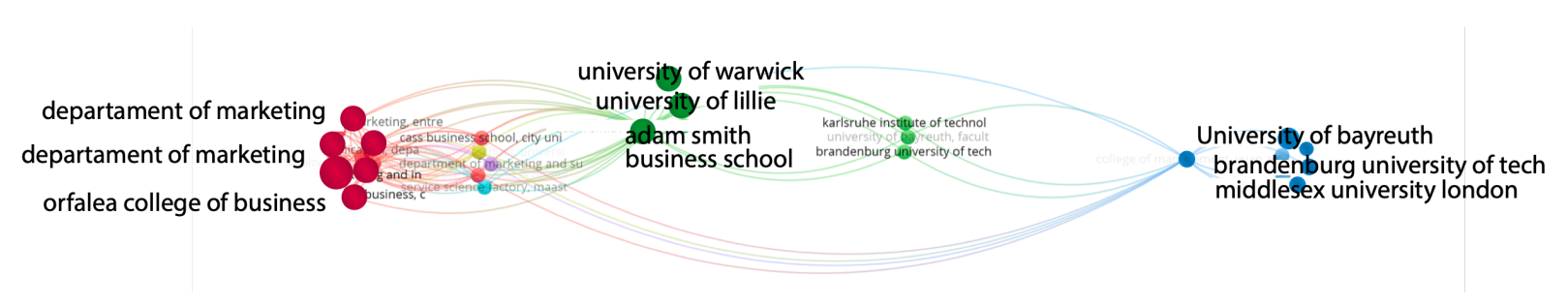

Gráfico 5 - Co-ocorrência de palavras-chave e os quatro grupos de proximidade. Fonte: acervo do autor.

Na rede bibliográfica por fonte de documento, destacam-se o Journal of Retailing and Consumer Services como grande centro das referências ligadas à área de moda, consumo, comunicação e tecnologia aplicada e o Lecture Notes in Computer Science, na área de tecnologia propriamente dita (Gráfico 6).

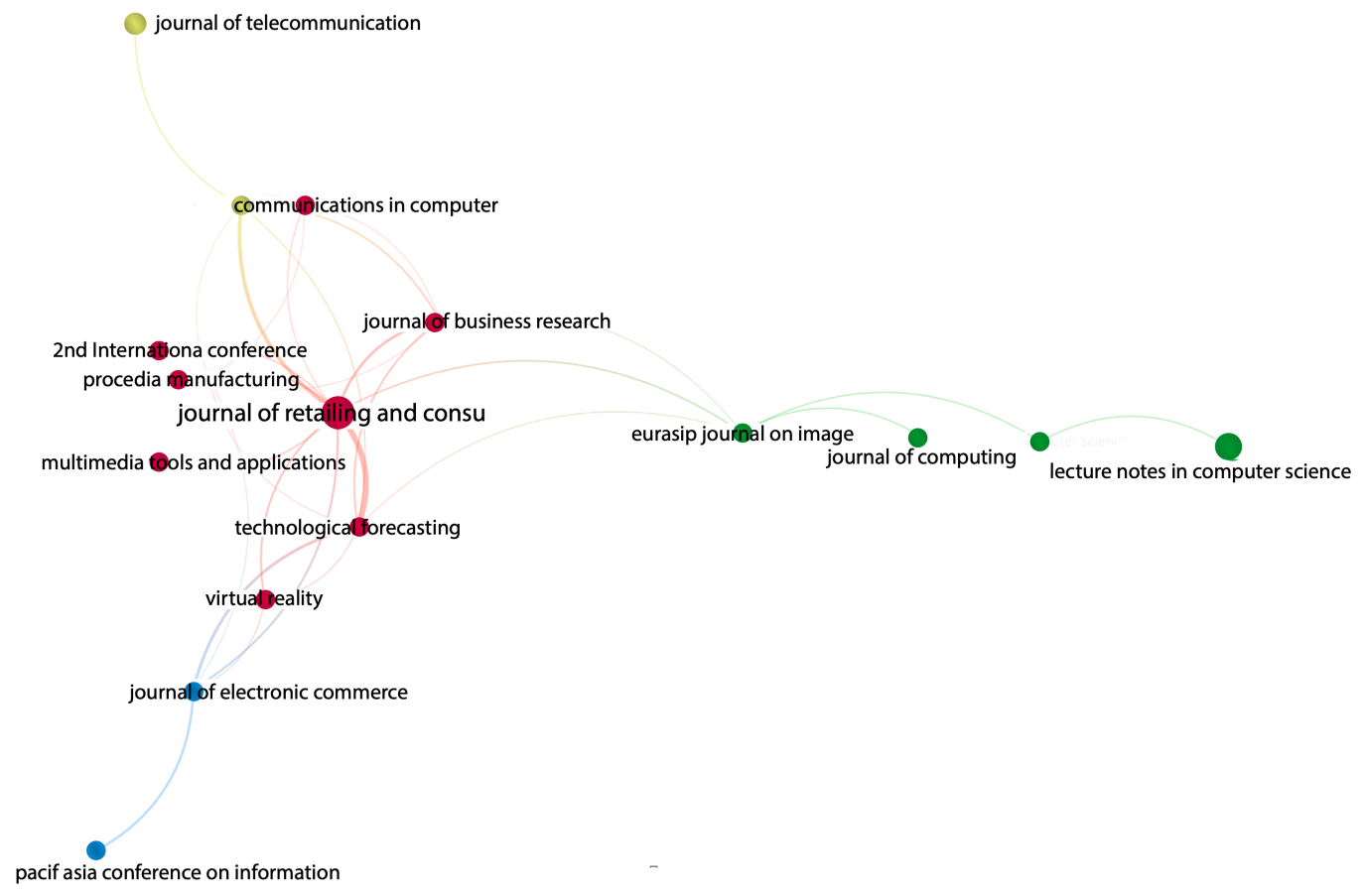

Gráfico 6 - Rede bibliográfica por fonte de documento. Fonte: acervo do autor. 
O Journal of Retailing and Consumer Services, ainda, é o periódico com a maior concentração de referências na seleção: seis. Este, editado pela Universiti Tecniakal Malaysia Melaka, possui citescore de 0,44 e seus eixos são engenharia elétrica e ciências da computação.

As demais referências estão em 14 periódicos e 8 conferências, todos com uma referência, à excessão de Lecture Notes In Computer Science Including Subseries Lecture Notes In Artificial Intelligence And Lecture Notes In Bioinformatics, International Conference on Augmented Reality, Virtual Reality and Computer Graphics, com duas referências cada. Apenas uma tese foi selecionada.

A maioria dos periódicos em que estes foram publicados se concentram em duas categorias estudos do consumidor (marketing, varejo e serviços) e tecnologia (ciências da computação e engenharia). Há poucas publicações, como Multimedia e Tools Applications e Conference on Human Factors in Computing Systems, que estão mais próximas à área do design, sendo este uma matéria presente em praticamente todos os documentos, mas não como tema central. Dessa forma, os campos de estudo que mais abordam o tema são computação, marketing, engenharia e matemática.

A revisão revelou, também, dez instituições com duas publicações na área pesquisada nos últimos cinco anos, sendo:

- Universidade Estadual Politécnica da California, Estados Unidos;

- Universidade Yuan Ze, Taiwan;

- Universidade Nacional Tsing Hua University, China;

- Universidade Maastricht, Holanda;

- Universidade de Londres, Inglaterra;

- Universidade Tecnológica de Brandenburg, Alemanha;

- Universidade de Bayreuth, Alemanha;

- Universidade de New South Wales, Austrália;

- Universidade de Massachussetts, Estados Unidos;

- S P Jain School of Global Management, Emirados Árabes.

$\mathrm{Na}$ análise do conjunto no Start, também foi possível observar para que classe de usuários a implementação estudada foi desenvolvida. Trinta e quatro documentos estudaram objetos destinados ao público-final. Em duas referências o público dos objetos era interno das empresas que as desenvolveram e apenas em uma referência, o objeto foi desenhado para uso em feira ou evento.

Quanto aos objetivos das pesquisas, onze documentos descrevem a implementação de tecnologia de realidade aumentada em catálogos virtuais de moda; vinte e três fazem uma análise de implementações do mesmo escopo e onze descrevem o uso da tecnologia pesquisada em uma diversidade de situações. O número superior ao total de referências se dá pelo fato de algumas referências possuírem mais de um objetivo (descrevendo e analisando uma tecnologia, por exemplo).

No domínio do protótipo, o desktop foi a plataforma utilizada em 28 das 37 referências da seleção, tendo apenas 8 documentos estudado objetos com dispositivos móveis como suporte e apenas uma referência se debruçou sobre objetos de estudo que utilizam ambas as plataformas.

Foram catalogadas 18 referências com o objeto estudado simulando roupas. Apenas em duas referências o objeto de estudo simula calçados. Acessórios foram simulados em 10 referências. Os demais documentos estudaram objetos que utilizaram a realidade aumentada em contextos múltiplos. Ficou demonstrado, também, a predominância do input de dados visuais na interação entre usuário e protótipo: todas as referências trabalham nessa modalidade. Seis artigos discorreram sobre objetos que utilizaram, além do input visual, periféricos, como joysticks e mouses. Dados gestuais foram citados em apenas um estudo e nenhum documento utilizou aplicações que fazem uso de dados sonoros.

Quanto aos métodos de avaliação utilizados, foram encontrados oito modelos, sendo que a falta de padronização e, em alguns casos, detalhamento, dificultou o entendimento de alguns procedimentos utilizados. Abaixo, as principais ocorrências:

- Questionário com usuários (descrição genérica): Sete referências;

- Framework: Duas referência;

- TAM (Technology Acceptance Model): Uma referência;

- Análise comparativa: Uma referência;

- Entrevista, vídeo e análise online do aplicativo e observação participativa: Uma referência;

- Análise heurística: Uma referência;

- Fleischer's Technology-Organization-Environment model (TOE): Uma referência;

- Escala Likert: Uma referência. 
É importante ressaltar um ponto em que a revisão sistemática não conseguiu apresentar resultados: dificuldades de implementação da tecnologia. Apenas dois documentos apontaram tal dado:

- Virtual Glasses Try-on Based on Large Pose Estimation: Usando apenas a câmera do computador desktop, a tecnologia registra apenas duas dimensões do ambiente, não reconhecendo a profundidade. Assim, os óculos são simulados pela tecnologia em frente do resto do usuário, o que pode parecer um erro em posições laterais de cabeça;

- A Cloud Service Framework for Virtual Try-On of Footwear in Augmented Reality: A tecnologia estudada não funcionou em tempo real, necessitando de um tempo de processamento do computador antes de ser exibida para o usuário.

O último problema notado foi a identificação da tecnologia de Realidade Aumentada utilizada, pois 14 referências não discorreram sobre tal ponto nas soluções que abordam. Nas demais, diferentes documentos abordaram diferentes contextos: alguns se debruçaram sobre o software e outros descreveram aspectos de implementação ou relação com o usuário. Ainda assim, os termos mais citados são realidade aumentada baseada em marcadores (3 referências) e Microsoft Kinect (3 referências).

\section{Considerações finais}

Esta revisão sistemática da literatura buscou referências que discorrem sobre uso de realidade aumentada em catálogos virtuais de moda. Para tanto utilizou as bases Elsevier Scopus, Science Direct, ISI Web of Science, Scielo e ProQuest, agrupando 2048 referências que, após processo de seleção, resultou em 37 documentos que atendem aos critérios postos no protocolo. A extração dos dados de tais referências revelou relações e respondeu às perguntas também aqui propostas.

O Journal of Retailing and Consumer Services revelou-se o periódico mais importante para o tema e o International Conference on Augmented Reality, Virtual Reality and Computer Graphics, o evento mais relevante. Os autores Pantano E., Beck M. e Rese A. trabalham nos diversos aspectos que englobam essa revisão e são citados em artigos em todas as perspectivas encontradas; Ylm M. Y., Atalar M. e Erra U. lidam comumente com questões tecnológicas (programação e hardware) e Baier D., Scholz J., Cruz E. e Wang C. com aspectos ligados ao marketing.

Os principais grupos de pesquisa, instituições e países trabalhando com o tema são: Academy of Arts \& Design, Tsinghua University, Beijin; Departamento de Computação e Ciências Tecnológicas da Tsinghua University, Beijin; Adam Smith Business School, Brandenburg University of Technology - Cadeira de Marketing e Inovação, Inglaterra; Karlsruhe Institute of Technology, Alemanha; Orfalea College of Business, IAE Lille School, França; University of Bayreuth; Alemanha.

Quanto aos objetivos de pesquisa das 37 referências selecionadas na revisão sistemática, 11 delas descrevem uma implementação de tecnologia de realidade aumentada em catálogos virtuais de moda; 23 fazem análise de implementações e outras 3 descrevem usos de tecnologia de Realidade Aumentada em catálogos virtuais de moda.

Dos objetos de pesquisa analisados, implementados ou descritos em tais referências, 28 são aplicações desenvolvidas para computadores desktop, 8 para suportes móveis (celulares e tablets) e apenas uma referência se debruçou sobre objetos projetados tanto para dispositivos móveis quanto para desktops. Tais objetos ainda, podem ser divididos pelo tipo de produto que simulam, sendo que 18 destes trabalhavam com roupas; 10 com acessórios (óculos, brincos, pulseiras, relógios etc), 7 com ítens de mais de uma categoria e apenas dois objetos simulam exclusivamente calçados.

Os inputs de dados, ainda nos objetos de estudo das referências são predominantemente visuais: todas as referências utilizam essa entrada de dados. Apenas 6 objetos fazem uso de periféricos (controles, joysticks etc); um faz uso de captura e interpretação de gestos e nenhum utiliza inputs sonoros.

\section{Agradecimentos}

Agradecemos ao departamento de pós-graduação em Design da UFSC, ao CNPq e aos laboratórios Hiperlab (UFSC) e Laboratório de Ergonomia (UDESC). 
A Systematic Literature Review on the use of augmented reality in the users' experience with virtual catalogs of products.

Abstract: This article presents a systematic review of the literature on quality of use of reality increases in the experience of users with virtual catalogs of fashion products in the last 5 years. The objectives were: to point out the relevance curve of the theme, main journals and institutions dealing with technology, research objectives, platforms used, types of simulation, modes of interaction and technologies used, evaluation methods and the main difficulties. 37 references were cataloged and these demonstrate the current state of the area: the increase in publications in the period; the Journal of Retailing and Consumer Services as a major center linked to the area of fashion, consumer behavior, communication and applied technology and the Lecture Notes in Computer Science, in technology itself; the main occurrences of keywords; leading researchers; the difficulty in identifying evaluation methods and lack of standardization in technology nomenclatures, among other discoveries.

Keywords: Systematic literature review; Augmented reality; Interface; Virtual fashion catalogs.

\section{Referências bibliográficas}

ALTARTEER, Samar et al. Development and Heuristic Evaluation of Semi-immersive Hand-Gestural Virtual Reality Interface for Luxury Brands Online Stores. Lecture Notes In Computer Science, [s.l.], p.464-477, 2017. Springer International Publishing. http://dx.doi.org/10.1007/978-3-319-60928-7_39.

ATALAR, Mustafa; OZCAN, Mahmut. New augmented reality application in E-commerce and M-commerce. 2017 International Conference On Computer Science And Engineering (ubmk), [s.l.], p.1-5, out. 2017. IEEE. http://dx.doi.org/10.1109/ubmk.2017.8093403.

BECK, Marie; CRIÉ, Dominique. I virtually try it ... I want it ! Virtual Fitting Room: A tool to increase on-line and off-line exploratory behavior, patronage and purchase intentions. Journal Of Retailing And Consumer Services, [s.l.], v. 40, p.279-286, jan. 2018. Elsevier BV. http://dx.doi.org/10.1016/ j.jretconser.2016.08.006.

BONNIN, Gaël. The roles of perceived risk, attractiveness of the online store and familiarity with AR in the influence of AR on patronage intention. Journal Of Retailing And Consumer Services, [s.l.], v. 52, p.101938-101941, jan. 2020. Elsevier BV. http://dx.doi.org/10.1016/j.jretconser.2019.101938.

CHANDRA, Shalini; KUMAR, Karippur Nanda. EXPLORING FACTORS INFLUENCING ORGANIZATIONAL ADOPTION OF AUGMENTED REALITY IN E-COMMERCE:: EMPIRICAL ANALYSIS USING TECHNOLOGYORGANIZATION- ENVIRONMENT MODEL. Journal Of Electronic Commerce Research. Long Beach, p. 237-265. ago. 2018.

CHU, Chih-hsing et al. A Cloud Service Framework for Virtual Try-On of Footwear in Augmented Reality. Journal Of Computing And Information Science In Engineering, [s.l.], v. 19, n. 2, p.0210021-0210027, 4 fev. 2019. ASME International. http://dx.doi.org/10.1115/1.4042102.

COCKTON, G. Usability Evaluation. In: SOEGAARD, Mads; DAM, Rikke Friis (Eds.). The Encyclopedia of Human-Computer Interaction, 2. ed. Aarhus, Denmark: The Interaction Design Foundation. Disponível em https://www.interaction-design.org/ encyclopedia/usability_evaluation.html. Acesso em: 1 de mai. 2019.

CRUZ, Edmanuel et al. An augmented reality application for improving shopping experience in large retail stores. Virtual Reality, [s.I.], v. 23, n. 3, p.281-291, 24 fev. 2018. Springer Science and Business Media LLC. http://dx.doi.org/10.1007/s10055-018-0338-3. 
DACKO, Scott G.. Enabling smart retail settings via mobile augmented reality shopping apps. Technological Forecasting And Social Change, [s.l.], v. 124, p.243-256, nov. 2017. Elsevier BV. http:// dx.doi.org/10.1016/j.techfore.2016.09.032.

ELRADI, Mahgoub et al. A 3D e-Commerce Applications Development Model: A Systematic Literature Review. Journal Of Telecommunication, Electronic And Computer Engineering. Kuala Lumpur, p. 27-33. abr. 2017.

ERRA, Ugo; SCANNIELLO, Giuseppe; COLONNESE, Valerio. Exploring the effectiveness of an augmented reality dressing room. Multimedia Tools And Applications, [s.I.], v. 77, n. 19, p.25077-25107, 20 fev. 2018. Springer Science and Business Media LLC. http://dx.doi.org/10.1007/ s11042-018-5758-2.

FENG, Zhuming; JIANG, Fei; SHEN, Ruimin. Virtual Glasses Try-on Based on Large Pose Estimation. Procedia Computer Science, [s.I.], v. 131, p.226-233, 2018. Elsevier BV. http://dx.doi.org/10.1016/ j.procs.2018.04.207.

HILKEN, Tim et al. Augmenting the eye of the beholder: exploring the strategic potential of augmented reality to enhance online service experiences. Journal Of The Academy Of Marketing Science, [s.l.], v. 45, n. 6, p.884-905, 18 maio 2017. Springer Nature. http://dx.doi.org/10.1007/ s11747-017-0541-x.

Tim et al. Making omnichannel an augmented reality: the current and future state of the art. Journal Of Research In Interactive Marketing, [s.l.], v. 12, n. 4, p.509-523, 8 out. 2018. Emerald. http://dx.doi.org/10.1108/jrim-01-2018-0023.

HÖLH, Wolfgang. Interactive Ambient with Opens-Source Software: 3D Walkthroughs and Augmented Reality for Architects with Blender 2.43, DART 3.0 and ARToolkit 2.72. Springer-Verlag: Viena, 2009, $239 \mathrm{p}$.

HUANG, Tseng-lung; LIAO, Shuling. A model of acceptance of augmented-reality interactive technology: the moderating role of cognitive innovativeness. Electronic Commerce Research, [s.l.], v. 15, n. 2, p.269-295, 12 nov. 2014. Springer Nature. http://dx.doi.org/10.1007/s10660-014-9163-2.

HUANG, Tseng-lung. Psychological mechanisms of brand love and information technology identity in virtual retail environments. Journal Of Retailing And Consumer Services, [s.l.], v. 47, p.251-264, mar. 2019. Elsevier BV. http://dx.doi.org/10.1016/j.jretconser.2018.11.016.

JAVORNIK, Ana et al. Revealing the Shopper Experience of Using a 'Magic Mirror' augmented reality make-up application. Proceedings Of The 2016 Acm Conference On Designing Interactive Systems - Dis '16, [s.l.], p.1-2, 2016. ACM Press. http://dx.doi.org/10.1145/2901790.2901881.

KUMAR, Karippur Nanda et al. FACTORS INFLUENCING ADOPTION OF AUGMENTED REALITY TECHNOLOGY FOR E-COMMERCE. In: PACIS 2016: Proceedings of the 20th Pacific Asia Conference on Information Systems. ISBN: 9789860491029. Association for Information Systems (2016).

LIBERATI, Nicola. The Emperor's New Augmented Clothes. Digital Objects as Part of the Every Day. Multimodal Technologies And Interaction, [s.l.], v. 1, n. 4, p.26-38, 23 out. 2017. MDPI AG. http://dx.doi.org/10.3390/mti1040026.

LIU, Yejun et al. Magic Mirror. Proceedings Of The 2016 Acm On Multimedia Conference - Mm '16, [s.I.], p.680-683, 2016. ACM Press. http://dx.doi.org/10.1145/2964284.2970928.

MAHONY, Stephen O'. A Proposed Model for the Approach to Augmented Reality Deployment in Marketing Communications. Procedia - Social And Behavioral Sciences, [s.l.], v. 175, p.227-235, fev. 2015. Elsevier BV. http://dx.doi.org/10.1016/j.sbspro.2015.01.1195.

MANGIARUA, Nahuel et al. Templates Framework for the Augmented Catalog System. Communications In Computer And Information Science, [s.I.], p.267-276, 2019. Springer International Publishing. http://dx.doi.org/10.1007/978-3-030-20787-8_19.

MCLEAN, Graeme; WILSON, Alan. Shopping in the digital world: Examining customer engagement through augmented reality mobile applications. Computers In Human Behavior, [s.l.], v. 101, p.210-224, dez. 2019. Elsevier BV. http://dx.doi.org/10.1016/j.chb.2019.07.002. 
MIELL, Sophie L.. Enabling the Digital Fashion Consumer through Gamified Fit and Sizing Experience Technologies. 2018. 374 f. Tese (Doutorado) - Curso de Philosophy In Textiles Design, Fashion \& Management, Faculty Of Science And Engineering, The University Of Manchester, Manchester, 2018.

OHTA, Masaya et al. [POSTER] Mixed-Reality Store on the Other Side of a Tablet. 2015 Ieee International Symposium On Mixed And Augmented Reality, [s.l.], p.192-193, set. 2015. IEEE. http://dx.doi.org/10.1109/ismar.2015.60.

OSAMURA, Kazuki et al. Proposal of Product Navigation Interface and Evaluation of Purchasing Motivation. Proceedings Of The Interactive Surfaces And Spaces On Zzz - Iss '17, [s.I.], p.458-461, 2017. ACM Press. http://dx.doi.org/10.1145/3132272.3135931

PANTANO, Eleonora; RESE, Alexandra; BAIER, Daniel. Enhancing the online decision-making process by using augmented reality: A two country comparison of youth markets. Journal Of Retailing And Consumer Services, [s.I.], v. 38, p.81-95, set. 2017. Elsevier BV. http://dx.doi.org/10.1016/j.jretconser.2017.05.011.

PANTANO, Eleonora; SERVIDIO, Rocco. Modeling innovative points of sales through virtual and immersive technologies. Journal Of Retailing And Consumer Services, [s.I.], v. 19, n. 3, p.279-286, maio 2012. Elsevier BV. http://dx.doi.org/10.1016/j.jretconser.2012.02.002.

PLOTKINA, Daria; SAUREL, Hélène. Me or just like me? The role of virtual try-on and physical appearance in apparel M-retailing. Journal Of Retailing And Consumer Services, [s.I.], v. 51, p.362-377, nov. 2019. Elsevier BV. http://dx.doi.org/10.1016/j.jretconser.2019.07.002.

RESE, Alexandra et al. How augmented reality apps are accepted by consumers: A comparative analysis using scales and opinions. Technological Forecasting And Social Change, [s.l.], v. 124, p.306319, nov. 2017. Elsevier BV. http://dx.doi.org/10.1016/j.techfore.2016.10.010.

ROUSE, Margaret. Augmented reality. 2016. Disponível em <http://whatis.techtarget.com/definition/augmented-reality-AR>. Acesso em: 28 abr. 2019.

SAAKES, Daniel et al. Mirror Mirror. Proceedings Of The 2016 Chi Conference On Human Factors In Computing Systems - Chi '16, [s.l.], p.6058-6063, 2016. ACM Press. http://dx.doi.org/ $10.1145 / 2858036.2858282$.

SCHOLZ, Joachim; DUFFY, Katherine. We ARe at home: How augmented reality reshapes mobile marketing and consumer-brand relationships. Journal Of Retailing And Consumer Services, [s.I.], v. 44, p.11-23, set. 2018. Elsevier BV. http://dx.doi.org/10.1016/j.jretconser.2018.05.004.

SCHOLZ, Joachim; SMITH, Andrew N.. Augmented reality: Designing immersive experiences that maximize consumer engagement. Business Horizons, [s.I.], v. 59, n. 2, p.149-161, mar. 2016. Elsevier BV. http://dx.doi.org/10.1016/j.bushor.2015.10.003.

SMINK, Anne R. et al. Try online before you buy: How does shopping with augmented reality affect brand responses and personal data disclosure. Electronic Commerce Research And Applications, [s.l.], v. 35, p.100854-100864, maio 2019. Elsevier BV. http://dx.doi.org/10.1016/ j.elerap.2019.100854.

STOYANOVA, Jasmina et al. Comparison of consumer purchase intention between interactive and augmented reality shopping platforms through statistical analyses. 2015 International Symposium On Innovations In Intelligent Systems And Applications (inista), [s.I.], p.1-2, set. 2015. IEEE. http://dx.doi.org/10.1109/inista.2015.7276727.

VENTURA, Felipe. Os primeiros experimentos com ARKit, plataforma de realidade aumentada da Apple. 2017. Disponível em <https://tecnoblog.net/217741/experimentos-arkit-apple/>. Acessado em: 28 de abr. De 2019.

YIM, Mark Yi-cheon; PARK, Sun-young. "I am not satisfied with my body, so I like augmented reality (AR)". Journal Of Business Research, [s.l.], v. 100, p.581-589, jul. 2019. Elsevier BV. http://dx.doi.org/10.1016/j.jbusres.2018.10.041. 
YIM, Mark Yi-cheon; CHU, Shu-chuan; SAUER, Paul L.. Is Augmented Reality Technology an Effective Tool for E-commerce? An Interactivity and Vividness Perspective. Journal Of Interactive Marketing, [s.I.], v. 39, p.89-103, ago. 2017. Elsevier BV. http://dx.doi.org/10.1016/j.intmar.2017.04.001.

WANG, Chao-hung; CHIANG, Yi-chen; WANG, Mao-jiun. Evaluation of an Augmented Reality Embedded On-line Shopping System. Procedia Manufacturing, [s.I.], v. 3, p.5624-5630, 2015. Elsevier BV. http://dx.doi.org/10.1016/j.promfg.2015.07.766.

WELIVITA, Anuradha et al. Virtual Product Try-On Solution for E-Commerce Using Mobile Augmented Reality. Lecture Notes In Computer Science, [s.I.], p.438-447, 2017. Springer International Publishing. http://dx.doi.org/10.1007/978-3-319-60922-5_34.

ZHANG, Boping. Augmented reality virtual glasses try-on technology based on iOS platform. Eurasip Journal On Image And Video Processing, [s.I.], v. 2018, n. 1, p.1-19, 27 nov. 2018. Springer Nature. http://dx.doi.org/10.1186/s13640-018-0373-8. 